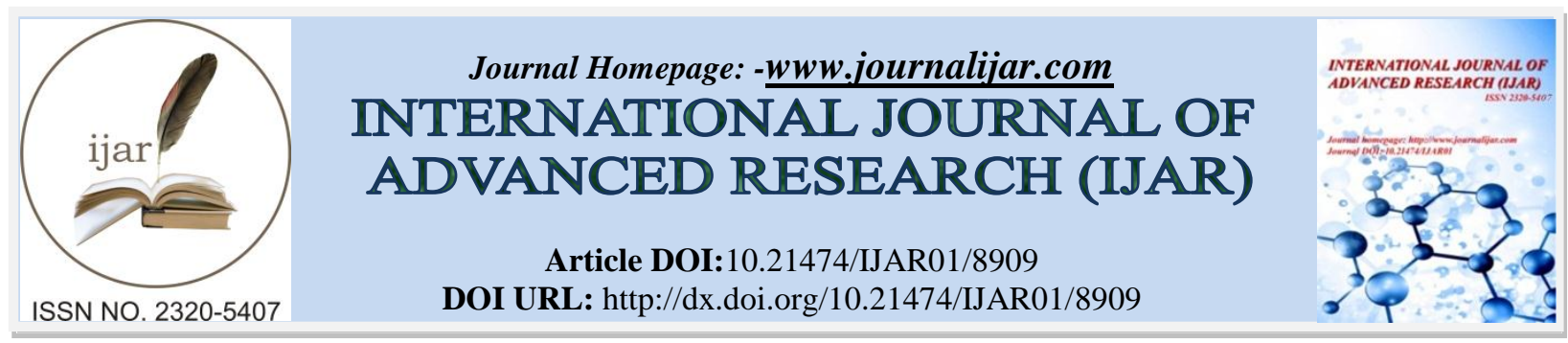

RESEARCH ARTICLE

\title{
BONE MARROW EXAMINATION IN VISCERAL LEISHMANIASES; A STUDY IN A TERTIARY CARE HOSPITAL.
}

\author{
Nuzhat samoon ${ }^{1}$, Irfan Hussain Bhat ${ }^{3}$, Aijaz Muzamil ${ }^{1}$, Sabiya Paddar $^{2}$, Tabassum ${ }^{2}$ and Huzaifa Nazier ${ }^{1}$. \\ 1. senior resident, Dept of Pathology, SKIMS, Soura. \\ 2. PG residents, Dept of Pathology, SKIMS, Soura. \\ 3. Post MCH senior resident, Dept of Neurosurgery.SKIMS, Soura.
}

\section{Manuscript Info}

\section{Manuscript History}

Received: 12 February 2019

Final Accepted: 14 March 2019

Published: April 2019

Key words:-

Bone marrow examination, visceral

leishmaniase, Sporadic.

\begin{abstract}
Background: Visceral Leishmaniasis a vector borne parasitic disease is endemic in many parts of the world. It is a chronic febrile ailment caused by Leishmania Donovani (LD). More than three hundred million people living in the endemic areas are at high risk and fourteen million are living with the disease. The leishmaniasis is not endemic but sporadic in our state.

Methods: This study was conducted over a period of 10 years in the department of Pathology and haematology, SKIMS Soura, focusing on the morphology of bone marrow aspirate and biopsy obtained from patients. The aspirate was smeared on glass slides, fixed with alcohol and stained with Giemsa stain. Bone marrow biopsy was fixed in formalin and stained with $\mathrm{H} \& \mathrm{E}$

Results : In our study we had total no of 9 patients as the disease is sporadic in our state.Male to female ratio was 2:1 with age range from 1 year old to 19 year old and mean age of 10 years .

Conclusion: Bone marrow aspiration and biopsy, although an invasive procedure, gives direct microscopic diagnosis of visceral Leishmaniasis and may be considered where indicated.
\end{abstract}

Copy Right, IJAR, 2019,. All rights reserved.

\section{Introduction:-}

Visceral Leishmaniasis (also known as Kala Azar), is a vector borne chronic febrile ailment caused by Leishmania Donovani (abbreviated as LD bodies) after the bite of sand fly having this parasite.(1) According to an estimate, more than 300 hundred million people living in the endemic areas are at high risk, while another 14 million are living with the disease. (2)The disease is endemic in many parts of the world including South East Asia. (3)On the public health importance of this parasitic infection. WHO accepted for the public health importance of this infection and proposed for these strategies (a) training program on diagnosis and case management of leishmaniasis; (b) setting up a harmonized regional surveillance system; (c) creating a connecting network of experts and (d) promoting international commitment of disease management. (4) It mostly affects children less than twelve years age (5) but cases have been reported in adults as well $(6,7)$

Corresponding Author:-Nuzhat samoon.

Address:-senior resident, Dept of Pathology, SKIMS, Soura. 


\section{Material \&methods:-}

This study was conducted over a period of 10 years at the department of Pathology SKIMS Soura, focusing on the morphology of bone marrow aspirate and biopsy obtained from patients. The marrow aspirate was smeared on glass slides, fixed with alcohol and stained with giemsa stain. The bone marrow biopsy was fixed in formalin and after decalcification was stained with haematoxylin and eosin stain.

\section{Results:-}

In our study we had total no of 9 patients as the disease is sporadic in our state.Male to female ratio was 2:1 with age range from 1 year old to 19 year old and mean age of 10 years. Patients presented with fever, anemia, weight loss and hepatosplenomegaly.

Bone marrow aspiration (Figure 1\&2) and biopsy (Figure 3) revealed amastigote forms of the parasite intraas well as extracellularly.

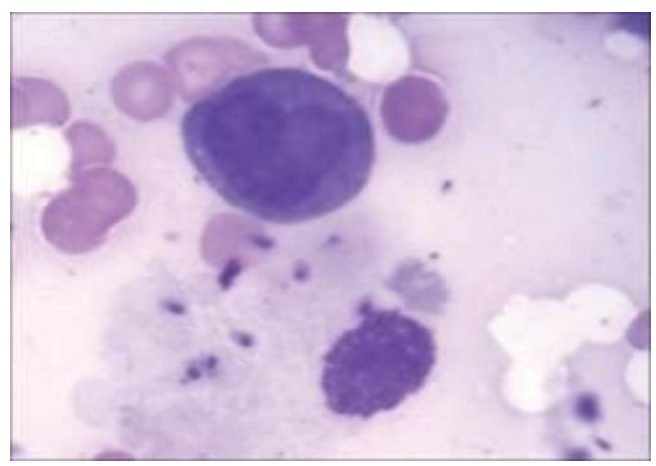

Figure 1:-Photomicrograph of bone marrow aspiration smear shows intra as well as extracellular LD bodies.( Geimsa, Oil immersion )

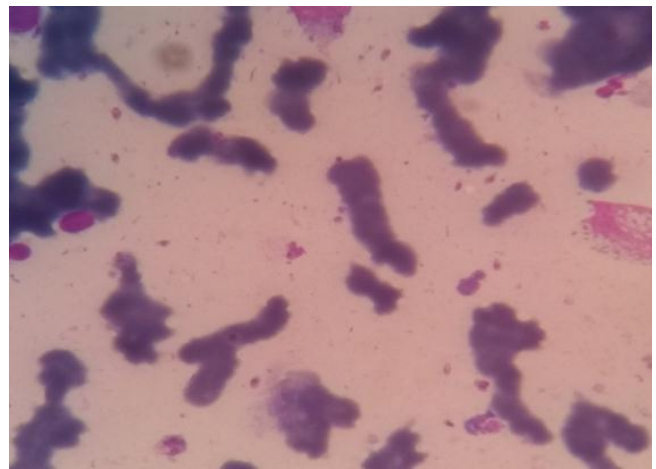

Figure 2:-Photomicrograph of bone marrow aspiration smear shows extracellular LD bodies. (Geimsa, Oil immersion )

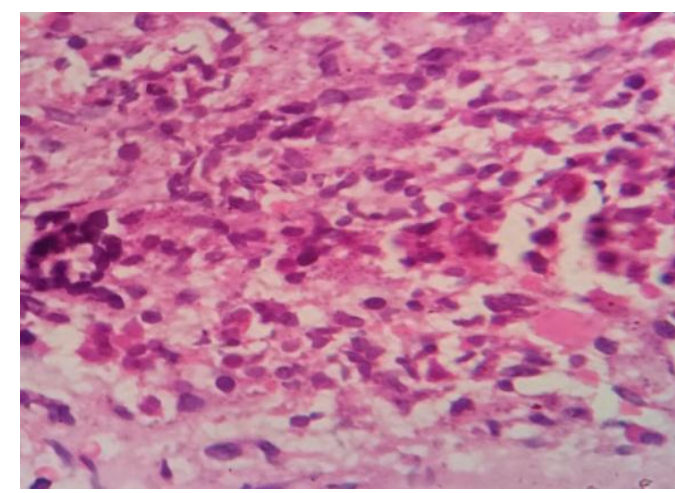

Figure 3:-Photomicrograph of bone marrow biopsy section show marrow element with dispersed macrophages filled with LD bodies. (H\&E, 400X) 


\section{Discussion:-}

Leishmanioses are a group of parasitic infections that affect human beings and other mammals. They are caused by different species of trypanosomatids of the genus Leishmania. $(8,9)$ Bone marrow examination is an authentic method of diagnosis of visceral Leishmaniasis. Both Intra-histiocytic and Extra-histiocytic LD bodies were seen. Megaloblastic change, erythroid hyperplasia and dyserythropoiesis were other significant findings.(10) Hypercellular marrow, increased plasma cells, granulomas, haemo-phagocytosis and gelatinous transformation of marrow were reported in some studies.(11) Bone marrow examination has been proposed as a technique of choice for establishing the parasitologic diagnosis of visceral Leishmaniasis.(12) Considering VL, the typical symptomatic triad, anemia, fever-enlarged spleen, may be not completely fulfilled in all cases however was seen in almost all cases of our study. The diagnosis of visceral form is generally derived by the demonstration of amastigotes of the leishmanial parasite(s) in the aspirated fluid from the bone marrow, the spleen, and rarely from the lymph nodes, or the liver (13). Pancytopenia was seen in half of the patients in our study. Berentsen et al said that the identification of the parasites in bone marrow biopsy should be performed in all pancytopenic patients with fevers of unknown origin and had the previous history of visiting to the Mediterranean, the endemic area of VL (13).

\section{Conclusion:-}

Bone marrow examination, although an invasive procedure, gives direct microscopic diagnosis of visceral Leishmaniasis and may be considered where indicated.

\section{Conflict of interest:}

None

\section{Funding:}

None

\section{Refrences:-}

1. Rab MA, Frame IA, Evans DA. The role of dogs in the epidemiology of human visceral leishmaniasis in northern Pakistan. Trans R Soc Trop Med Hyg 1995;89(6):612-5.

2. Pace D. Leishmaniasis. J Infect 2014;69(Suppl 1):S10-8.

3. Meheus F, Boelaert M. The burden of Visceral Leishmaniasis in South Asia. Trop Med Int Health 2010;15 Suppl 2:1-3.

4. Postigo JA. Leishmaniasis in the World Health Organization Eastern Mediterranean Region. Int J Antimicrob Agents 2010; 36(Suppl 1): S62-S65.

5. Savoia D. Recent updates and perspectives on Leishmaniasis. J Infect Dev Ctries 2015;9(6):588-96.

6. El Jeri HK, Harzallah A, Barbouch S, Bacha MM, Kheder R, Turki S, et al. Visceral Leishmaniasis in Adults with Nephropathy. Saudi J Kidney Dis Transpl 2017;28(1):95-101

7. Lahlou H, Filali AB, Alami M, Amrani M, Berrady R, Rabhi S, et al. Visceral leishmaniasis in 26 HIV negative adults. BMC Res Notes 2011;4:389.

8. Murray HW, Berman JD, Davies CR, Saravia NG. Advances in leishmaniasis. Lancet 2005; 366: 1561-1577.

9. Gallego M. Emerging parasitic zoonoses: leishmaniosis. Rev Sci Tech 2004; 23: 661-676.

10. Raina S, Raina RK. Hematological Profile of Newly Diagnosed Patients with Visceral Leishmaniasis from a Non-Endemic Hilly Region of India. Ann Clin Pathol 2017;5(1):1100.

11. Dhingra KK, Gupta P, Saroha V, Setia N, Khurana N, Singh T. Morphological findings in bone marrow biopsy and aspirate smears of visceral Kala Azar: a review. Indian J Pathol Microbiol 2010;53(1):96-100.

12. da Silva Mr, Stewart JM, Costa CH. Sensitivity of bone marrow aspirates in the diagnosis of visceral leishmaniasis. Am J Trop Med Hyg 2005;72(6):811-4.

13. Singh S, Sivakumar R. Recent advances in the diagnosis of leishmaniasis. J Postgrad Med 2003; 49: 55-60. 